\title{
Association with Social Jetlag and Time Preference of Migraine Attack: A Pilot Study
}

\author{
Ji Hye Shin ${ }^{1}$, Hee-Jin $\operatorname{Im}^{2}$ \\ ${ }^{1}$ Department of Neurology, Korea Univerisity Anam Hospital, Seoul, \\ ${ }^{2}$ Department of Neurology, Hangang Sacred Heart Hospital, Hallym University Medical Center, Seoul, Korea
}

Received May 14, 2019

Revised June 12, 2019

Accepted June 17, 2019

Address for correspondence

Hee-Jin Im, MD, MMSc

Department of Neurology,

Hangang Sacred Heart Hospital,

Hallym University

Medical Center, 7-12

Budnaru-ro, Youngdeungpo-gu,

Seoul 07247, Korea

Tel: +82-2-2639-5720

Fax: +82-2-2639-5029

E mail: coolere@naver.com
Objectives: To evaluate association between presence of time preference of headache attack and social jetlag and circadian preference in patients with migraine with or without aura. Methods: A total of 50 patients of migraine with or without aura who visited for the first time at a single tertiary medical center were examined and interviewed about their sleep pattern on weekdays and weekends and whether they have time preference of headache attack during a day or not. Frequency of headache and measurement for disability of headache such as six-item Headache Impact Test and other sleep profiles such as sleep duration, sleep quality, daytime sleepiness, and insomnia severity were obtained by a booklet of questionnaire. Results: In this pilot study, the time preference of migraine attack during a day was present in $42 \%$ among the participants. In the cross-sectional analysis, the group of presence of time preference of attack showed earlier circadian preference (1:36 am $\pm 282 \mathrm{~min}$ vs. $4: 24 \mathrm{am} \pm 186 \mathrm{~min}, p=0.02)$ and lower social jetlag than the other group $(0.4 \pm 0.8$ hour vs. $1.4 \pm 2.2$ hour, $p=0.04)$. Other demographic characteristics and frequency and severity of headache was comparable between groups. Conclusions: Considering an individual circadian preference might be a strategy in integrated management of migraine.

J Sleep Med 2019;16(1):48-52

Key Words: Time preference, Chronotype, Social jetlag, Migraine.

\section{Introduction}

The relationship between primary headache, especially migraine, and sleep has been emerging for over a century. One of pathognomic characteristics of migraine is relapsing, remittent, pleomorphic patterns. Migraine attacks have a periodic tendency of menstrual, seasonal and circadian rhythm, suggesting a predisposed mechanism based on chronobiology. ${ }^{1}$ Several evidences emphasized on involvement of hypothalamus in circadian alteration in migraine.

A prospective longitudinal study has been demonstrated that migraine attacks in the early morning especially between 4:00 and 9:00 a.m. implicating relationship with rapid eye movement sleep. ${ }^{2}$ In the other side of view, early morning is preferable time for people with early chronotype who have advanced phase of sleep and wake cycle (called as 'morning lark'). Migraineurs has been related with early chronotype compared to normal controls in a large clinic-based study [odds ratio (OR) 2.42, confidence interval (CI) 1.58-3.69]. ${ }^{3}$

When individually entrained chronotype is mismatched with social cycle, all behaviors and emotions can be affected and loose the balance of daily life. Misalignment between internal and social clock to satisfy social accomplishment is one of key concerning factors in chronobiology in modern society, and which is called social jetlag (SJL). A number of negative aspects of circadian derangement in physical and mental illness are recently emerged interest. Concerning the SJL bothers our daily life, not only for physical or mental unhealthiness, one of most common primary headache, migraine, can be affected because it is known to be under circadian propensity.

The aim of this study was to evaluate the association time preference of headache attack and SJL in migraineurs.

\section{Methods}

\section{Participants}

This study was a single clinic center based cross-sectional and descriptive survey. We included 50 patients with migraine with or without aura who visited for headache clinic in department of neurology of Korea University Anam Hospital, 
Seoul, Republic of Korea from March 2017 to March 2018. Patients who were not treated with prescription analgesics were enrolled and all the participants were interviewed clinically, and examined neurologically and physically by one neurologist. The study design was approved by the Institutional Review Board of Korea University Anam Hospital (No. 2019A N0214). All participants provided written informed consent.

\section{Clinical assessment of headache}

The diagnosis of migraine with and without aura was made based on the diagnostic criteria of the International Classification of Headache Disorders, the third edition. Participants were asked to fill out the booklet of questionnaire at their first visit to the clinic for headache. Preferential time attack of headache during day is asked by the question as follows: "On average, do you think your headaches tend to be concentrated time during the day?" The response is obtained as either 'yes' or 'no.'

The participants with migraine were asked whether the frequency of their migraine daily, weekly, monthly, or <monthly. The severity of headache was reported by two parameters. One is subjective scoring of visual analog scale (VAS). Patients recorded pain intensity on a $100 \mathrm{~mm}$-horizontal line. The left end score of the VAS ' 0 ' represented 'no pain' and the right end score of VAS ' 10 ' represented 'most severe pain. The other severity variable is subjective grading whether subjects are suffering from daily activity due to their headache or not.

The six-item Headache Impact Test (HIT-6) has been validated for adverse disabling impact of migraine in clinical practice. ${ }^{4}$ A total HIT-6 score ranges from 36 to 78 , where a higher score indicates a greater impact of headache on the daily life. To measure level of disability due to headache, we adopted HIT-6 scale to the participants. Neuroimaging were performed for confirmation of no structural abnormality for their headache by either brain computed tomography or magnetic resonance imaging.

\section{Variables about sleep profiles}

Information of sleep onset time and sleep end time on weekdays (workdays) and weekends (free days) was obtained. The average weekly sleep duration is calculated as weighted value depending on weekdays and weekends: $(5 \times$ sleep duration on workdays $+2 \times$ sleep duration on free days)/7. We estimated the midpoint of sleep on free days corrected for sleep extension on free days (MSFsc) as an indicator of chronobiological preference, so called chronotype as Roenneberg has suggested in 2004. ${ }^{5-7}$ It was calculated as follows: MSFsc= midpoint of sleep on free days $-0.5 \times$ (sleep duration on free days-average weekly sleep duration)

SJL was defined as discrepancy between internal and social clock result from chronotype-driven preference and social obligation for sleep times for sleep times. Conventionally it was defined as absolute difference of mid-sleep time on free days (weekends) and word days (weekdays) since Wittmann et al. suggested as follows: SJL=|mid sleep on free days-mid sleep on word days|. ${ }^{8}$

Sleep quality was assessed using the Pittsburgh Sleep Quality Index, with poor sleep quality as a score $\geq 5 .{ }^{9}$ Excessive daytime sleepiness (EDS) was defined when the Epworth Sleepiness Scale was higher than $10 .{ }^{10}$ Insomnia was evaluated using the Insomnia Severity Index in which a score of more than 14 was the cut-off value for moderate-to-severe clinical insomnia. ${ }^{11}$

\section{Other variables}

To evaluate the presence and severity of depressed mood, the Patient Health Questionnaire-9 depression scale was used, which is composed of nine items. Each item is one of the nine criteria on which the diagnosis of Diagnostic and Statistical Manual of Mental Disorders-IV (DSM-IV) depressive disorder is made, and each is scored from 0 to 3 . A total score $\geq 10$ is defined as the presence of depressed mood. ${ }^{12}$ Anxiety exists when the Beck's Anxiety Inventory score $\geq 22$, which consists of 21 symptoms and each symptom is scored as 1 when present. ${ }^{13}$ Body mass index (BMI, $\left.\mathrm{kg} / \mathrm{m}^{2}\right)$ was calculated from self-reported height $(\mathrm{cm})$ and body weight $(\mathrm{kg})$.

\section{Statistical analysis}

The primary aim was to evaluate difference of chronotype and SJL according to presence of time preference of attack. For group comparisons between subjects with and without presence of time preference of attack, a 2-tailed Student's ttest or chi-square test was used to for continuous or categorical variables, respectively. The data analysis was performed using SPSS version 24.0 (IBM Corp., Armonk, NY, USA), and $p<0.05$ indicated statistical significance.

\section{Results}

\section{Characteristics of participants with migraine}

In 50 subjects, the average age was $37.6 \pm 16.4$ years and female were 39 patients (78.0\%) (Table 1). Migraine with aura was present in $48.0 \%$ (24 patients). As the survey was performed at the first visit for their headache in a neurology clinic of tertiary medical center, the burden and severity of headache they reported was over than moderate. The average HIT- 6 score related disability was $61.4 \pm 10.6$ points and severe degree of disability (more than 50 points of HIT-6 score) was present in $84 \%$ of study population. Subjective VAS score was $6.8 \mathrm{~cm}$ among $10 \mathrm{~cm}$ maximum. Nineteen patients (38.0\%) reported that they were suffering their daily activity due to 
Table 1. Baseline characteristics in total participants $(n=50)$

\begin{tabular}{|c|c|}
\hline Characteristics & mean \pm SD or $\mathrm{n}(\%)$ \\
\hline Age (year) & $37.6 \pm 16.4$ \\
\hline Female sex & $39(78.0)$ \\
\hline Education ( $\geq 12$ education-year) & $26(53.1)$ \\
\hline Job (yes) & $35(70.0)$ \\
\hline BMI $\left(\mathrm{kg} / \mathrm{m}^{2}\right)$ & $22.8 \pm 2.9$ \\
\hline Alcohol use & $13(26.5)$ \\
\hline Regular exercise & $10(20.0)$ \\
\hline Current smoking & $6(12.2)$ \\
\hline Visual Aura (+) & $24(48.0)$ \\
\hline \multicolumn{2}{|l|}{ Frequency } \\
\hline$<$ Monthly & $5(10.4)$ \\
\hline Monthly & $9(18.8)$ \\
\hline Weekly & $20(41.7)$ \\
\hline Daily & $14(29.2)$ \\
\hline \multicolumn{2}{|l|}{ Severity } \\
\hline VAS score $(\mathrm{cm})$ & $6.8 \pm 1.8$ \\
\hline Disturbance of daily activity $(+)$ & $19(38.0)$ \\
\hline HIT-6 score & $61.4 \pm 10.6$ \\
\hline Severe headache (HIT- $6 \geq 50)$ & $42(84.0)$ \\
\hline BAI score & $15.6 \pm 13.3$ \\
\hline Moderate anxiety $(\mathrm{BAI} \geq 22)$ & $13(27.1)$ \\
\hline PHQ-9 depression scale score & $9.4 \pm 6.5$ \\
\hline Depressive mood & $19(38.0)$ \\
\hline
\end{tabular}

SD: standard deviation, n: number, BMI: body mass index, VAS: visual analog scale, HIT-6: six-item Headache Impact Test, , BAI: Beck Anxiety Inventory, PHQ-9: Patient Health Questionnaire-9

headache. Most patients have headache in frequency at least weekly (41.7\%) and 29.2\% has daily headache.

\section{Association with SJL/chronotype and time preference of migraine attack}

Less than half $(42 \%, 21$ patients) participants reported presence of time preference of their migraine attack (Table 2). There is no significant difference of frequency or severity of headache between groups whether there is presence of time preference of attack or not. Overall sleep profiles such as sleep duration on average weekly, weekdays and weekends, insomnia severity, sleep quality were comparable between groups. The group with presence of time preference of migraine attack had earlier circadian preference than the other group (1:36 am $\pm 282 \mathrm{~min}$ vs. $4: 24 \mathrm{am} \pm 186 \mathrm{~min}, p=0.022$ ). And SJL was higher in the lack of time preference group which was later chronotype $(0.4 \pm 0.8$ hour vs. $1.4 \pm 2.2$ hour, $p=0.040)$. Additionally, the degree of anxiety and daytime sleepiness was higher in the lack of time preference group but proportion of moderate anxiety and EDS was comparable between groups.

\section{Discussion}

In this pilot study, we demonstrate an association between presence of time preference of migraine attack and earlier circadian preference in a relatively small sized clinic-based sample. While its small sample size, the demographic characteristics of the participants of this study, women in thirty's was consistent with the prevalent those of migraine with other large-sized clinical studies.

Transient and episodic hypothalamic dysfunction in its known pathophysiology, ${ }^{14}$ migraine is related to be circadian biology which is regulated by suprachiasmatic nucleus of the hypothalamus. Association with migraine and seasonal variation has been proved and discussed in a few evidences. Few decades ago, one Norwegian study demonstrated patients with migraine with aura showed marked seasonal preference with more attacks in the light season and time of peak attack was May 21. This light seasonal preponderance is robust in female with migraine with aura. ${ }^{1}$

Our study stands for tendency of early chronotype in migraineurs with time preference of headache attack. Predominant attacks in the early morning especially between 4:00 and 9:00 am has been reported before. ${ }^{2}$ This means clear relationship of timing as well as intensity of light exposure. The known prevalent time interval in the previous study was actually active time zone for the early chronotype, who prefers to wake in the early morning, called 'morning lark.' One recent large Netherland study has suggested migraineurs more often tended to be early chronotype than normal controls (OR 2.42, CI 1.10-2.61) and less half (40.2\%) subjects has reported on early morning attack frequently. ${ }^{3}$

Sleep timings are controlled when social and internal clocks are well synchronized. The disruption of this balance is called SJL. It has been associated predominantly with the late chronotype who have undesirable patterns with social routines which usually starts work at morning, and had quantitatively positive relationship with MSFsc. ${ }^{8}$ It is plausible that the tendency of early chronotype in the group of time preference of migraine attack could also link to lower SJL than other group in our study (Table 2). However, more various confounding factor need to be considered with real-world extended study the exploration of the stroke association about the chronotype such as age and light exposures.

SJL represents discrepancy between biological and social clock, it is known to be typically higher in the evening chronotype in most social routine time of school or work are set in the morning. A lot of evidences reported that later chronotype experienced adverse physical and mental outcomes such 
Table 2. Comparison between groups with the presence of time preference of migraine attack and not $(n=50)$

\begin{tabular}{|c|c|c|c|}
\hline Characteristics & Time preference $(+)(n=21)$ & Time preference $(-)(n=29)$ & $p$ value \\
\hline Age (year) & $41.7 \pm 16.2$ & $34.6 \pm 16.2$ & 0.133 \\
\hline Female sex & $14(66.7)$ & $25(86.2)$ & 0.166 \\
\hline Daily headache & $4(19.0)$ & $10(37.0)$ & 0.174 \\
\hline Disturbance on daily activity $(+)$ & $8(38.1)$ & $11(37.9)$ & 0.991 \\
\hline VAS score $(\mathrm{cm})$ & $6.8 \pm 1.8$ & $6.9 \pm 1.8$ & 0.920 \\
\hline HIT-6 score & $61.8 \pm 11.4$ & $61.1 \pm 10.1$ & 0.826 \\
\hline Severe headache (HIT-6 $\geq 50)$ & $18(85.7)$ & $24(82.8)$ & 0.778 \\
\hline BAI score & $11.2 \pm 7.4$ & $17.7 \pm 15.9$ & $0.028^{*}$ \\
\hline Moderate anxiety $(\mathrm{BAI} \geq 22)$ & $3(14.3)$ & $10(37.0)$ & 0.078 \\
\hline PHQ-9 depression scale score & $7.8 \pm 5.7$ & $10.6 \pm 15.7$ & 0.160 \\
\hline Depressive mood & $6(31.6)$ & $12(48.0)$ & 0.242 \\
\hline SD average (hour) & $6.4 \pm 1.3$ & $6.6 \pm 1.4$ & 0.462 \\
\hline SD weekday (hour) & $6.0 \pm 1.5$ & $5.7 \pm 1.7$ & 0.573 \\
\hline SD weekend (hour) & $6.6 \pm 1.7$ & $6.9 \pm 2.1$ & 0.641 \\
\hline MSFsc & $1: 36 \mathrm{am} \pm 282 \mathrm{~min}$ & $4: 24 \mathrm{am} \pm 186 \mathrm{~min}$ & $0.022^{*}$ \\
\hline Social jetlag (hour) & $0.4 \pm 0.8$ & $1.4 \pm 2.2$ & $0.040^{*}$ \\
\hline PSQI score & $8.5 \pm 2.3$ & $9.8 \pm 4.6$ & 0.219 \\
\hline Poor sleep quality & $19(90.5)$ & $23(79.3)$ & 0.441 \\
\hline ESS & $5.3 \pm 3.7$ & $7.7 \pm 6.0$ & 0.090 \\
\hline Excessive daytime sleepiness & $1(4.8)$ & $9(31.0)$ & $0.031^{*}$ \\
\hline ISI score & $8.6 \pm 6.2$ & $10.2 \pm 6.6$ & 0.404 \\
\hline Moderate-to-severe insomnia & $4(19.0)$ & $8(27.6)$ & 0.485 \\
\hline
\end{tabular}

Data presented as mean \pm standard deviation or number (\%) $p$ values from Students' t-test or Fisher's/chi-square test. ${ }^{*} p<0.05$. VAS: visual analog scale, HIT-6: six-item Headache Impact Test, BAI: Beck Anxiety Inventory, PHQ-9: Patient Health Questionnaire-9, SD: sleep duration, MSFsc: midpoint of sleep on free days corrected for sleep extension on free days, PSQI: Pittsburgh Sleep Quality Index, ESS: Epworth Sleepiness Scale, ISI: Insomnia Severity Index

as obesity and poor psychological wellbeing. ${ }^{6,15}$ In lack of time preference group, they showed more severe degree of anxiety and daytime sleepiness compared with the other group. It would be explained those in the group had later chronotype and higher SJL in consequence. BMI was little bit higher in lack of time preference group than the other group $(23.3 \pm 2.6$ vs. $22.2 \pm 3.2, p=0.34$ ) though significantly week.

The strength of our study is one of few studies focusing on chronobiology especially about SJL related with migraine and assessment for association between time preference of attack and early circadian preference which is consistent with points of other relevant studies. Also an interesting point our study is that severity and disability of migraine of our subject was moderate severe and its impact on their daily life was quite strong who visits the tertiary medical center in Republic of Korea for headache. The limitation of our study is a single center based small-sized pilot study. The study design is cross-sectional and it needs more multidirectional analysis for detailed explanation. Moreover, the key interested factor, the presence of time preferential attack is not an objective, but a subjective variable. It needs more objective or time-detailed assessment of time zone of preferential time in extended analysis.

Migraine is common but complex and multifactorial disorder. There is a clear association of periodicity and frequency of migraine related to sleep. ${ }^{16}$ To cope with these aspects, clinicians need to be consider time preference of attacks and chronotype whether to decide to treat their patients for prevention of frequent headache.

\section{Conflicts of Interest}

The authors have no potential conflicts of interest to disclose

\section{Acknowledgments}

This study was supported by the Research Grant of Korean Sleep Research Society in 2018.

\section{ORCID iDs}

Ji Hye Shin

Hee-Jin Im

https://orcid.org/0000-0002-3536-7748

https://orcid.org/0000-0002-8979-6521

\section{Author Contributions}

Conceptualization: Hee-Jin Im. Data curation: Ji Hye Shin. Formal anal- 
ysis: Hee-Jin Im. Funding acquisition: Hee-Jin Im. Investigation: Hee-Jin Im. Methodology: Hee-Jin Im. Writing_original draft: Hee-Jin Im. Writing_-review \& editing: Hee-Jin Im.

\section{REFERENCES}

1. Alstadhaug KB, Bekkelund S, Salvesen R. Circannual periodicity of migraine? Eur J Neurol 2007;14:983-988.

2. Fox AW, Davis RL. Migraine chronobiology. Headache 1998;38:436-441.

3. van Oosterhout W, van Someren E, Schoonman GG, et al. Chronotypes and circadian timing in migraine. Cephalalgia 2018;38:617-625.

4. Yang M, Rendas-Baum R, Varon SF, Kosinski M. Validation of the Headache Impact Test $\left(\right.$ HIT- ${ }^{\mathrm{TM}}$ ) across episodic and chronic migraine. Cephalalgia 2011;31:357-367.

5. Roenneberg T, Kuehnle T, Pramstaller PP, et al. A marker for the end of adolescence. Curr Biol 2004;14:R1038-R1039.

6. Wittmann M, Paulus M, Roenneberg T. Decreased psychological wellbeing in late 'chronotypes' is mediated by smoking and alcohol consumption. Subst Use Misuse 2010;45:15-30.

7. Juda M, Vetter C, Roenneberg T. Chronotype modulates sleep duration, sleep quality, and social jet lag in shift-workers. J Biol Rhythms 2013;28:141-151.
8. Wittmann M, Dinich J, Merrow M, Roenneberg T. Social jetlag: misalignment of biological and social time. Chronobiol Int 2006;23:497-509.

9. Buysse DJ, Reynolds CF 3rd, Monk TH, Berman SR, Kupfer DJ. The Pittsburgh Sleep Quality Index: a new instrument for psychiatric practice and research. Psychiatry Res 1989;28:193-213.

10. Johns MW. A new method for measuring daytime sleepiness: the Epworth sleepiness scale. Sleep 1991;14:540-545.

11. Bastien CH, Vallières A, Morin CM. Validation of the Insomnia Severity Index as an outcome measure for insomnia research. Sleep Med 2001;2:297-307.

12. Kroenke K, Spitzer RL. The PHQ-9: a new depression diagnostic and severity measure. Psychiatric Annals 2002;32:509-515.

13. Beck AT, Epstein N, Brown G, Steer RA. An inventory for measuring clinical anxiety: psychometric properties. J Consult Clin Psychol 1988; 56:893-897.

14. Overeem S, van Vliet JA, Lammers GJ, Zitman FG, Swaab DF, Ferrari MD. The hypothalamus in episodic brain disorders. Lancet Neurol 2002;1:437-444.

15. Baron KG, Reid KJ, Kern AS, Zee PC. Role of sleep timing in caloric intake and BMI. Obesity 2011;19:1374-1381.

16. Rains JC, Poceta JS, Penzien DB. Sleep and headaches. Curr Neurol Neurosci Rep 2008;8:167-175. 